\title{
CONTROLE ROBUSTO DE ROBÔS MÓVEIS EM FORMAÇÃO SUJEITOS A FALHAS
}

\author{
Adriano A. G. Siqueira* \\ siqueira@sc.usp.br
}

\author{
Marco H. Terra ${ }^{\dagger}$ \\ terra@sc.usp.br
}

\author{
Tatiane B. R. Francisco ${ }^{\dagger}$ \\ tatibrf@sel.eesc.usp.br \\ *Departamento de Engenharia Mecânica \\ ${ }^{\dagger}$ Departamento de Engenharia Elétrica \\ Universidade de São Paulo - 13566-590, São Carlos, SP, Brasil
}

\begin{abstract}
Robust and Fault Tolerant Control of Wheeled Mobile Robots In Formation

This paper deals with robust and fault tolerant controls of wheeled mobile robots (WMRs) in formation. The robust approach is based on output feedback nonlinear $\mathcal{H}_{\infty}$ control. The fault tolerant approach is based on output feedback $\mathcal{H}_{\infty}$ control of Markovian jump linear systems to ensure stability of the formation when one of the robots is lost during the coordinated motion. The coordination is performed with the robots following a leader, and any robot of the formation can assume the leadership randomly. The mobile robots exchange informations according to a pre-specified communication directed graph (digraph).
\end{abstract}

KEYWORDS: Formation Control, Mobile Robots, Nonlinear $\mathcal{H}_{\infty}$ Control, Markovian Control.

\section{RESUMO}

Este artigo trata de controle robusto e controle tolerante a falhas de movimentos coordenados de robôs móveis com rodas. O controle robusto é baseado em controle $\mathcal{H}_{\infty}$ não linear por realimentação da saída. O controle

Artigo submetido em 16/04/2009 (Id.: 00993)

Revisado em 22/06/2009, 23/09/2009, 29/10/2009

Aceito sob recomendação do Editor Associado Prof. Alexandre Bazanella tolerante a falhas é baseado em controle $\mathcal{H}_{\infty}$ por realimentação da saída de sistemas lineares sujeitos a saltos Markovianos para garantir estabilidade da formação quando um dos robôs é perdido durante o movimento coordenado. A coordenação é realizada em função de um lider e qualquer robô da formação pode assumir a liderança de maneira aleatória. Os robôs móveis trocam informações de acordo com um grafo de comunicação direcionado (dígrafo), definido a-priori.

PALAVRAS-CHAVE: Controle de Formação, Robôs Móveis, Controle $\mathcal{H}_{\infty}$ Não Linear, Controle Markoviano.

\section{INTRODUÇÃO}

Diversas abordagens têm sido apresentadas na literatura para resolver problemas de controle de múltiplos robôs móveis, (Chaimowicz et al., 2004; Pereira et al., 2008). Em particular, vamos considerar neste artigo estratégias de controle para robôs móveis com rodas (RMRs) que seguem um líder, veja por exemplo (Desai et al., 1998) e (Tanner et al., 2004). Uma abordagem que utiliza teoria dos grafos foi proposta por (Fax and Murray., 2004) para o controle cooperativo de múltiplos sistemas lineares. A teoria dos grafos é uma importante ferramenta capaz de tratar adequadamente os problemas que envolvam comunicação de dados entre agentes do sistema, (Lafferriere et al., 2005; Olfati-Saber and Murray, 2002). 
Apesar do grande número de resultados que tem aparecido na literatura, não foram encontrados procedimentos que envolvam controle robusto de robôs móveis em formação sujeitos a alternância de líder. Também não foram encontrados sistemas tolerantes a falhas baseados nos modelos dinâmicos dos robôs para esse tipo de problema. Esses dois aspectos são tratados neste artigo.

$\mathrm{Na}$ primeira estratégia de controle formulada consideramos um conjunto de RMRs em formação onde os robôs mantêm constantes as suas posições e orientações relativas durante o movimento. Cada RMR fornece a sua informação de posição somente a um subconjunto do grupo, estabelecido pelo projetista através de um dígrafo. A partir das trajetórias desejadas geradas pelo controlador descrito em (Williams et al., 2005), projetamos um controlador $\mathcal{H}_{\infty}$ não linear com realimentação da saída que considera as características cinemáticas e dinâmicas dos robôs móveis em formação. O objetivo é aplicar o controle robusto para um grupo de RMRs que devem seguir uma determinada trajetória e se manter em formação mesmo quando há alternância de lider durante o movimento. Esta estratégia de controle é usada para rejeitar primeiramente determinadas incertezas paramétricas e perturbações externas em cada robô da formação. Também vale destacar a vantagem de se utilizar abordagens robustas utilizando os modelos dinâmicos de cada RMR quando o movimento coordenado ocorre em alta velocidade.

Na segunda estratégia de controle desenvolvemos um sistema tolerante a falhas para evitar instabilidade na formação dos robôs quando houver perda de um dos robôs durante o movimento. Com base na teoria de Markov desenvolvemos um modelo que representa as possíveis configurações de formação e considera as mudanças abruptas de configuração. O modelo é desenvolvido baseado em sistemas lineares sujeitos a saltos Markovianos (SLSM) (Siqueira et al., 2007). Para formular este modelo, as dinâmicas dos robôs são linearizadas em torno de pontos de operação. As probabilidades de ocorrência de falhas e dos robôs estarem em determinados pontos de linearização são definidas a priori e dependem de cada aplicação específica. Com o modelo proposto, o controlador $\mathcal{H}_{\infty}$ por realimentac̃ão de saída de SLSM desenvolvido em (de Farias et al., 2000) é utilizado para garantir a estabilidade da formação mesmo quando o sistema está sujeito a falha.

Este artigo está organizado da seguinte maneira: na Seção 2 são apresentados os procedimentos necessários para a geração das trajetórias desejadas, na Seção 3 é apresentado o controle cinemático dos robôs móveis, na Seção 4 é apresentado o controle robusto baseado na dinâmica dos robôs móveis, na Seção 5 é apresentada a estratégia de controle tolerante a falhas baseada nos modelos lineares sujeitos a saltos Markovianos dos robôs móveis com rodas e na Seção 6 são apresentados resultados de simulação referentes às duas estratégias de controle apresentadas.

\section{GERAÇÃO DAS TRAJETÓRIAS DE RE- FERÊNCIA}

Nesta seção é apresentado o controlador descentralizado proposto em (Williams et al., 2005) cuja finalidade é gerar as trajetórias de referência para que os RMRs se movimentem em formação rígida. Assume-se que $N$ robôs móveis participem do movimento coordenado sendo que a trajetória de referência de cada robô é representada no sistema de coordenadas inercial $(X, Y)$ pela seguinte equação de estado:

$$
\dot{x}_{i}=A_{v e h} x_{i}+B_{v e h} u_{i}, \quad i=1, \ldots, N, \quad x_{i} \in \mathbb{R}^{4}
$$

sendo que $x_{i}=\left[\begin{array}{llll}x_{r_{i}} & \dot{x}_{r_{i}} & y_{r_{i}} & \dot{y}_{r_{i}}\end{array}\right]^{T}$ representa as posições para o $i$-ésimo RMR e suas derivadas, e $u_{i}$ representa as entradas de controle. As matrizes $A_{v e h}$ e $B_{v e h}$, têm a seguinte forma:

$$
A_{\text {veh }}=\left(\begin{array}{cccc}
0 & 1 & 0 & 0 \\
0 & a_{22} & 0 & a_{24} \\
0 & 0 & 0 & 1 \\
0 & a_{42} & 0 & a_{44}
\end{array}\right), \quad B_{v e h}=\left(\begin{array}{cc}
0 & 0 \\
1 & 0 \\
0 & 0 \\
0 & 1
\end{array}\right)
$$

sendo $a_{i j}$ constantes relacionadas à trajetória desejada para a formação. A trajetória do líder pode ser alterada variando-se os elementos da matriz $A_{v e h}$. Por exemplo, se $a_{22}=a_{24}=a_{42}=a_{44}=0$, temos uma reta com inclinação dada pelas condições iniciais; se $a_{22}=a_{44}=0$ e $a_{24}=-k$ e $a_{42}=k$, com $k>0$, temos uma elipse. Esta variação pode ser realizada ao longo do tempo para se criar uma trajetória variável do líder. Os zeros nas colunas um e três de $A_{\text {veh }}$ são necessários para garantir a convergência dos robôs móveis para a formação (veja (Laferriere et al., 2004) Proposição 3.1 e (Veerman et al., 2005) Proposição 4.2).

O vetor $x=\left[\begin{array}{llll}x_{1}^{T} & x_{2}^{T} & \ldots & x_{N}^{T}\end{array}\right]^{T}$ descreve a combinação de todas as trajetórias dos $N$ robôs. Os vetores de posição e velocidade são definidos, respectivamente, por

$$
\begin{aligned}
& x_{p}=\left[\begin{array}{lll}
\left(x_{p}\right)_{1}^{T} & \ldots & \left(x_{p}\right)_{N}^{T}
\end{array}\right]^{T} \mathrm{e} \\
& x_{v}=\left[\begin{array}{lll}
\left(x_{v}\right)_{1}^{T} & \ldots & \left(x_{v}\right)_{N}^{T}
\end{array}\right]^{T},
\end{aligned}
$$

sendo $\left(x_{p}\right)_{i}=\left[\begin{array}{ll}x_{r_{i}} & y_{r_{i}}\end{array}\right]^{T}$ e $\left(x_{v}\right)_{i}=\left[\begin{array}{ll}\dot{x}_{r_{i}} & \dot{y}_{r_{i}}\end{array}\right]^{T}$. Assim, o vetor $x$ é reescrito como

$$
x=x_{p} \otimes\left(\begin{array}{l}
1 \\
0
\end{array}\right)+x_{v} \otimes\left(\begin{array}{l}
0 \\
1
\end{array}\right)
$$


sendo que $\otimes$ representa o Produto de Kronecker.

A definição de formação a ser considerada neste trabalho é caracterizada pelo vetor

$$
h=h_{p} \otimes\left(\begin{array}{l}
1 \\
0
\end{array}\right) \in \mathbb{R}^{4 N},
$$

sendo $h_{p}=\left[\begin{array}{lll}\left(h_{p}\right)_{1}^{T} & \ldots & \left(h_{p}\right)_{N}^{T}\end{array}\right]^{T}$ o vetor das posições descritas no sistema de coordenadas inercial $(X, Y)$ que definem a formação, sendo $\left(h_{p}\right)_{i}=\left[h_{x_{i}} h_{y_{i}}\right]$.

Os $N$ RMRs estão em formação $h$ no tempo $t$ se existirem vetores $q, w \in \mathbb{R}^{2}$, tais que,

$$
\left(x_{p}\right)_{i}(t)-\left(h_{p}\right)_{i}=q(t) \text { e }\left(x_{v}\right)_{i}(t)=w(t),
$$

para $i=1,2, \ldots, N$. Note que $q(t)=\left[q_{x}(t) q_{y}(t)\right]$ representa a distância entre a posição atual do robô $i$ e a posição do mesmo robô definida pela formação $h$. Esta distância deve ser a mesma para cada robô. Da mesma forma, $w$ representa a velocidade da formação, sendo que todos os robôs devem possuir a mesma velocidade.

Os RMRs convergem para a formação $h$ se existirem funções $q(),. w(.) \in \mathbb{R}^{2}$, tais que,

$$
\left(x_{p}\right)_{i}(t)-\left(h_{p}\right)_{i}-q(t) \rightarrow 0 \text { e }\left(x_{v}\right)_{i}(t)-w(t) \rightarrow 0,
$$

quando $t \rightarrow \infty$, para $i=1, \ldots, N$. A Figura 1 ilustra este conceito de formação, sendo representada apenas a análise com relação à coordenada $x$ do sistema de coordenadas inercial $(X, Y)$.

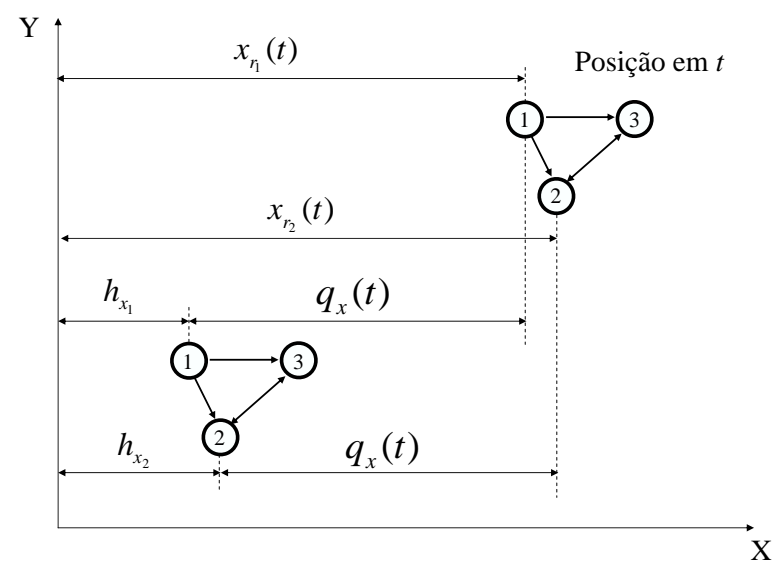

Figura 1: Representação de uma formação com três RMRs.

A topologia de comunicação entre os robôs móveis é representada por um dígrafo $\Gamma$ que captura a conectividade entre os robôs móveis. Cada vértice representa um robô e há uma aresta direcionada de um vértice ao outro se houver comunicação entre os robôs móveis. O robô móvel que envia a informação é considerado vizinho do robô que está recebendo a informação. O par $(i, j)$ pertence ao conjunto de arestas $\mathcal{E}$ se $i$ é vizinho de $j$. Para implementar o controle de formação, utiliza-se uma matriz Laplaciana que tem o objetivo de agrupar as informações que definem quais robôs se comunicam entre si.

Seja $\Gamma$ um dígrafo com conjunto de vértices $\mathcal{V}$ e conjunto de $\operatorname{arestas} \mathcal{E}$. A matriz de adjacência de $\Gamma$ é a matriz quadrada $Q$ com entradas

$$
q_{i j}=\left\{\begin{array}{ll}
1, & \text { se }(j, i) \in \mathcal{E}, \\
0, & \text { caso contrário. }
\end{array} \quad(i, j \in \mathcal{V}) .\right.
$$

A matriz de grau interno de $\Gamma$, definida como sendo diagonal, é denotada por $D$ e formada por

$$
d_{i i}=|\{j \in \mathcal{V}:(j, i) \in \mathcal{E}\}|, \quad(i \in \mathcal{V}) .
$$

Dado um dígrafo $\Gamma$, a matriz Laplaciana associada a ele é dada por (Brualdi and Ryser, 1991)

$$
L_{\Gamma}=D^{+}(D-Q)
$$

sendo $D$ uma matriz não invertível e o superescrito + denota pseudoinversa.

O robô considerado líder da formação é caracterizado por não receber nenhuma informação de outro robô móvel. Isto significa que os outros robôs são forçados a arranjar suas posições em resposta ao movimento desse robô (Lafferriere et al., 2005). Para que haja este líder, o dígrafo de comunicação é uma árvore. Trata-se de um dígrafo $\Gamma$ que não tem ciclos e admite um vértice $v$ (a raiz) tal que há um trajeto direcionado de $v$ a todos os outros vértices em $\Gamma$. Neste caso, a linha da matriz de adjacência $Q$ referente ao vértice do líder tem que ser zero.

As informações de cada robô móvel podem ser combinadas de tal forma que os RMRs devem ajustar seus movimentos com relacão ao líder da formação. As funções de saída $z_{i}$ podem ser calculadas pela média dos deslocamentos relativos (e velocidades relativas) da vizinhança dos respectivos robôs móveis como sendo (veja (Fax and Murray., 2004) e (Williams et al., 2005)) para maiores detalhes)

$$
z_{i}= \begin{cases}\frac{1}{\left|J_{i}\right|} \sum_{j \in J_{i}}\left(\left(x_{i}-h_{i}\right)-\left(x_{j}-h_{j}\right)\right), & \text { se }\left(\left|J_{i}\right| \neq 0\right) \\ 0 & \text { c.c. }\end{cases}
$$


para $i=1 \ldots N$, sendo que $J_{i}$ indica o número de vizinhos do $i$-ésimo robô. $\mathrm{O}$ vetor de saída $z$ pode ser escrito como $z=L(x-h)$ sendo $L=L_{\Gamma} \otimes I_{4}$ e $L_{\Gamma}$ a matriz direcionada Laplaciana do dígrafo $\Gamma$ (Williams et al., 2005).

Agrupando as equações para todos os RMRs em um único sistema no espaço de estado tem-se

$$
\begin{aligned}
& \dot{x}=A x+B u \\
& z=L(x-h)
\end{aligned}
$$

sendo $A=I_{N} \otimes A_{v e h}$ e $B=I_{N} \otimes B_{v e h}$. A existência de uma matriz de realimentação $F$ tal que a solução de

$$
\dot{x}=A x+B F L(x-h)
$$

converge para formação $h$ pode ser vista em (Williams et al., 2005). Este é um problema de estabilização envolvendo realimentação da saída. Considerando as estruturas de $A, B$ e $L$ tem-se $F$ da forma $F=I_{N} \otimes F_{v e h}$ (que é uma lei de controle descentralizada aplicada a todos os robôs móveis). Neste caso, pode-se reescrever a Eq. (2) como

$$
\dot{x}=A x+L_{\Gamma} \otimes B_{v e h} F_{v e h}(x-h) .
$$

Note que o conjunto de robôs móveis convergirá para uma dada formação $h$ se o Laplaciano do grafo direcionado $L_{\Gamma}$ possuir pelo menos dois autovalores nulos, (Lafferriere et al., 2005). Para obtermos o controlador, a matriz de realimentação, $F_{v e h}$, pode ser representada da seguinte forma

$$
F_{v e h}=\left[\begin{array}{cccc}
f_{1} & f_{2} & 0 & 0 \\
0 & 0 & f_{1} & f_{2}
\end{array}\right] .
$$

Em (Williams et al., 2005) mostra-se que as condições necessárias e suficientes para o sistema convergir para a formação são obtidas com $f_{1}<0$ e $f_{2}<0$.

A principal finalidade do controle de formação é encontrar a matriz de realimentação $F_{v e h}$ que garanta a convergência para a formação, dada a estrutura de $A_{v e h}$. Os elementos da matriz $A_{\text {veh }}$ são os parâmetros que definem a trajetória de referência para o robô líder. O controle mostrado acima gera as trajetórias que os demais RMRs devem realizar para entrar em formação com o líder. Estas curvas são utilizadas como trajetórias de referência para os robôs móveis, sendo consideradas como entradas para o controle cinemático descrito no próxima seção. $\mathrm{O}$ controle cinemático fornece, a partir das trajetórias de referência e das posições atuais do robôs, as posições e velocidades desejadas das rodas de cada robô, que por sua vez são utilizadas para realizar o controle baseado no modelo dinâmico dos robôs. A Figura 2 mostra o diagrama de blocos simplificado das estratégias de controle utilizadas neste trabalho.

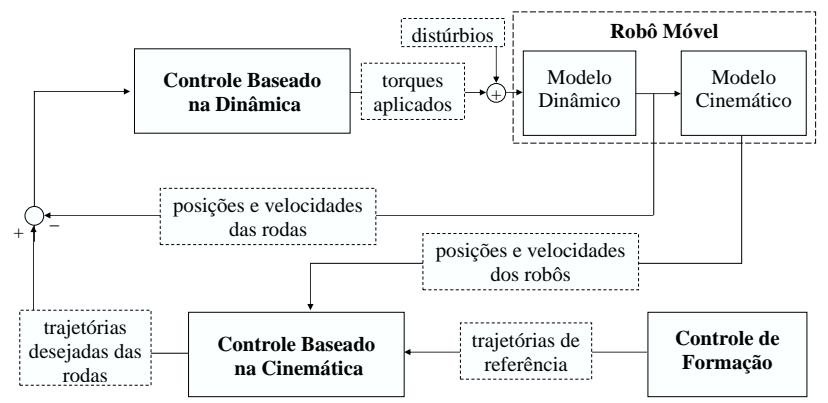

Figura 2: Diagrama de blocos simplificado das estratégias de controle.

\section{CINEMÁTICA DOS ROBÔS MÓVEIS}

Nesta seção é apresentado o controlador cinemático utilizado para garantir que os RMRs do tipo diferencial, considerados neste trabalho, acompanhem as trajetórias de referência geradas pelo controle de formação.

A geometria de cada robô é mostrada na Figura 3. Assume-se que todos os RMRs têm a mesma dimensão e que a roda passiva não influencia o comportamento cinemático e dinâmico do robô. Na Figura $3,(X, Y)$ representa o sistema de coordenadas inercial; $\left(X_{0}, Y_{0}\right)$ o sistema de cordenadas local; $a$ o comprimento do robô; $d$ a distância entre $P_{0}$ (centro do eixo das rodas atuadas) e $P_{c}$ (centro de massa); $b$ a distância entre uma roda atuada e o eixo de simetria do robô; $r$ o raio das rodas atuadas; $\alpha$ o ângulo (direção do robô) entre o eixo $X$ do referencial inercial e o eixo de simetria do robô no sentido horário e $\theta_{d}$ e $\theta_{e}$ são os deslocamentos angulares das rodas direita e esquerda, respectivamente.

Robôs móveis do tipo diferencial apresentam três restrições cinemáticas (Coelho and Nunes., 2003). Considerando que para este tipo de robô a velocidade em $P_{0_{i}}$ deve estar na direção do eixo de simetria (eixo $X_{0_{i}}$ ), tem-se a primeira restrição com relação a $P_{c_{i}}$, dada por

$$
\dot{y}_{c_{i}} \cos \alpha_{i}-\dot{x}_{c_{i}} \sin \alpha_{i}-d \dot{\alpha}_{i}=0, \quad i=1, \ldots, N
$$

sendo $\left(x_{c_{i}}, y_{c_{i}}\right)$ as coordenadas do centro de massa $P_{c_{i}}$ no sistema de coordenadas inercial e $\alpha_{i}$ o ângulo entre o eixo de simetria de cada robô da formação e o eixo $X$. As outras duas restrições estão relacionadas com a rotação das rodas, em outras palavras, as rodas atuadas 


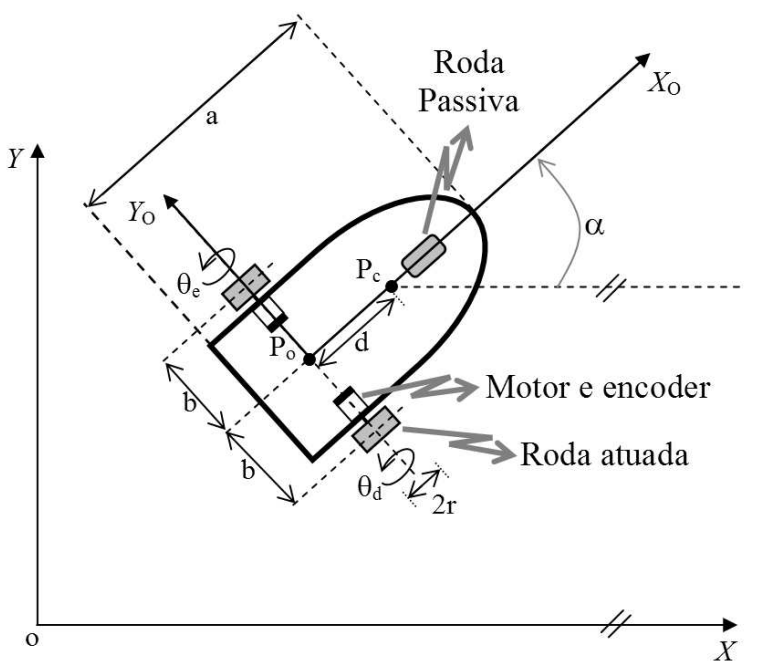

Figura 3: Robô móvel do tipo unicilo.

não deslizam

$$
\begin{aligned}
& \dot{x}_{c_{i}} \cos \alpha_{i}+\dot{y}_{c_{i}} \sin \alpha_{i}+b \dot{\alpha}_{i}-r \dot{\theta}_{d_{i}}=0 \\
& \dot{x}_{c_{i}} \cos \alpha_{i}+\dot{y}_{c_{i}} \sin \alpha_{i}-b \dot{\alpha}_{i}-r \dot{\theta}_{e_{i}}=0 .
\end{aligned}
$$

Definindo a coordenada generalizada $q_{1_{i}}=$ $\left[\begin{array}{lllll}x_{c_{i}} & y_{c_{i}} & \alpha_{i} & \theta_{d_{i}} & \theta_{e_{i}}\end{array}\right]^{T}$, as três restrições cinemáticas podem ser escritas na forma matricial como $R\left(q_{1_{i}}\right) \dot{q}_{1_{i}}=0$, sendo

$$
R\left(q_{1_{i}}\right)=\left[\begin{array}{ccccc}
-\sin \alpha_{i} & \cos \alpha_{i} & -d & 0 & 0 \\
-\cos \alpha_{i} & -\sin \alpha_{i} & -b & r & 0 \\
-\cos \alpha_{i} & -\sin \alpha_{i} & b & 0 & r
\end{array}\right] .
$$

A matriz $R\left(q_{1_{i}}\right)$ tem posto completo e pode ser expressa como $\left[R_{1}\left(q_{1_{i}}\right)\right.$ R $\left.R_{2}\right]$ de tal forma que $R_{1}\left(q_{1_{i}}\right)$ seja nãosingular e $S\left(q_{1_{i}}\right)=\left[\left(R_{1}^{-1}\left(q_{1_{i}}\right) R_{2}\right)^{T} I_{4}\right]^{T}$ esteja no espaço nulo de $R\left(q_{1_{i}}\right)$, i.e., $R_{1}\left(q_{1_{i}}\right) S\left(q_{1_{i}}\right)=0$. Então, encontra-se

$$
S\left(q_{1_{i}}\right)^{T}=\left[\begin{array}{cc}
c\left(b \cos \alpha_{i}-d \sin \alpha_{i}\right) & c\left(b \cos \alpha_{i}+d \sin \alpha_{i}\right) \\
c\left(b \sin \alpha_{i}+d \cos \alpha_{i}\right) & c\left(b \sin \alpha_{i}-d \cos \alpha_{i}\right) \\
c & -c \\
1 & 0 \\
0 & 1
\end{array}\right]
$$

sendo $c=\frac{r}{2 b}$. A equação cinemática é dada por

$$
\dot{q}_{1_{i}}(t)=S\left(q_{1_{i}}\right) \dot{q}_{2_{i}}(t)
$$

$\mathrm{Ou}$

$$
\begin{aligned}
\dot{x}_{c_{i}} & =c\left(b \cos \alpha_{i}-d \sin \alpha_{i}\right) \theta_{d_{i}}+c\left(b \cos \alpha_{i}+d \sin \alpha_{i}\right) \theta_{e_{i}} \\
\dot{y}_{c_{i}} & =c\left(b \sin \alpha_{i}+d \cos \alpha_{i}\right) \theta_{d_{i}}+c\left(b \sin \alpha_{i}-d \cos \alpha_{i}\right) \theta_{e_{i}} \\
\dot{\alpha}_{i} & =c\left(\theta_{d_{i}}-\theta_{e_{i}}\right) .
\end{aligned}
$$

sendo $q_{2_{i}}=\left[\begin{array}{ll}\theta_{d_{i}} & \theta_{e_{i}}\end{array}\right]^{T}$ o vetor das posições das rodas direita e esquerda do $i$-ésimo robô e $\dot{q}_{2_{i}}=\left[\begin{array}{ll}\dot{\theta}_{d_{i}} & \dot{\theta}_{e_{i}}\end{array}\right]^{T} \mathrm{o}$ vetor das velocidades angulares das rodas.

O controlador baseado na cinemática proposto por (Kanayama et al., 1990), fornece as velocidades angulares desejadas das rodas esquerda e direita, $\dot{q}_{2_{i}}^{d}$, tais que os RMRs acompanhem as trajetórias de referência geradas pelo controle de formação.

Considere o erro $q_{e_{i}}=\left[x_{e_{i}} y_{e_{i}} \alpha_{e_{i}}\right]^{T}$, entre a postura de referência $q_{r_{i}}=\left[\begin{array}{lll}x_{r_{i}} & y_{r_{i}} & \alpha_{r_{i}}\end{array}\right]^{T}$, obtida pela equação do controle de formação, (3), com

$$
\alpha_{r_{i}}=\operatorname{tg}^{-1}\left(\dot{y}_{r_{i}} / \dot{x}_{r_{i}}\right),
$$

e a postura atual de cada robô móvel na formação $q_{c_{i}}=$ $\left[\begin{array}{lll}x_{c_{i}} & y_{c_{i}} & \alpha_{c_{i}}\end{array}\right]^{T}$. As equações dos erros são dadas por

$$
\begin{aligned}
x_{e_{i}} & =\cos _{c_{i}}\left(x_{r_{i}}-x_{c_{i}}\right)+\sin \alpha_{c_{i}}\left(y_{r_{i}}-y_{c_{i}}\right), \\
y_{e_{i}} & =-\sin \alpha_{c_{i}}\left(x_{r_{i}}-x_{c_{i}}\right)+\cos \alpha_{c_{i}}\left(y_{r_{i}}-y_{c_{i}}\right), \\
\alpha_{e_{i}} & =\alpha_{r_{i}}-\alpha_{c_{i}} .
\end{aligned}
$$

O controle cinemático fornece as velocidades lineares $\left(v_{i}^{d}\right)$ e angulares $\left(\omega_{i}^{d}\right)$ desejadas dos RMRs, considerando o erro de postura e as velocidades lineares e angulares de referência dadas, respectivamente, por

$$
v_{r_{i}}=\sqrt{\left(\dot{x}_{r_{i}}\right)^{2}+\left(\dot{y}_{r_{i}}\right)^{2}} \text { e } \omega_{r_{i}}=\dot{\alpha}_{r_{i}} .
$$

sendo $\dot{x}_{r_{i}}$ e $\dot{y}_{r_{i}}$ obtidas pela equação do controle de formação, (3), e $\dot{\alpha}_{r_{i}}$ obtida derivando-se (6) com relação ao tempo.

Portanto, o controlador cinemático é dado por

$$
\begin{aligned}
v_{i}^{d} & =v_{r_{i}} \cos \left(\alpha_{e_{i}}\right)+K_{x} x_{e_{i}}, \\
\omega_{i}^{d} & =\omega_{r_{i}}+v_{r_{i}}\left(K_{y} y_{e_{i}}+K_{\alpha} \operatorname{sen} \alpha_{e_{i}}\right),
\end{aligned}
$$

sendo $K_{x}, K_{y}, K_{\alpha}$ constantes definidas pelo projetista.

O controle baseado na dinâmica considera as velocidades angulares desejadas das rodas de cada RMR, $\dot{q}_{2_{i}}^{d}$. Então, é necessário definir as seguintes relações de velocidades

$$
\dot{q}_{2_{i}}^{d}=\left[\begin{array}{c}
\dot{\theta}_{d_{i}}^{d} \\
\dot{\theta}_{e_{i}}^{d}
\end{array}\right]=\left[\begin{array}{cc}
1 / r & b / r \\
1 / r & -b / r
\end{array}\right]\left[\begin{array}{c}
v_{i}^{d} \\
\omega_{i}^{d}
\end{array}\right]
$$

sendo $\dot{\theta}_{d_{i}}^{d}$ and $\dot{\theta}_{e_{i}}^{d}$ as velocidades angulares desejadas das rodas direita e esquerda dos RMRs, respectivamente.

\section{REPRESENTAÇÃO QUASE-LPV DOS RMRS}

Na seção anterior estabelecemos a maneira como as trajetórias desejadas devem ser geradas para o controle de 
RMRs em formação, com base em informações cinemáticas dos robôs. Nesta seção vamos considerar os aspectos dinâmicos dos RMRs. Inicialmente será obtida uma representação quase-LPV (quase-Linear com Parâmetros Variantes) dos RMRs. Sistemas LPV são sistemas cujas matrizes dinâmicas variam continuamente conforme a variação no tempo de determinados parâmetros. Quando um sistema não linear é representado como um sistema LPV cujos parâmetros variantes correspondem aos estados ou parte dos estados, dizemos que temos uma representação quase-LPV daquele. Neste trabalho, o projeto de controle $\mathcal{H}_{\infty}$ com realimentação da saída para sistemas LPV descrito em (Apkarian and Adams, 1998) será utilizado para se obter um controlador baseado na dinâmica dos RMRs.

A equação dinâmica dos robôs móveis, usando a teoria de Lagrange, é descrita por Coelho and Nunes. (2003) como:

$$
M\left(q_{1_{i}}\right) \ddot{q}_{1_{i}}+C\left(q_{1_{i}}, \dot{q}_{1_{i}}\right) \dot{q}_{1_{i}}=E \tau_{i}-R\left(q_{1_{i}}\right)^{T} \lambda_{i}
$$

sendo $\lambda_{i}=\left[\begin{array}{lll}\lambda_{1_{i}} & \lambda_{2_{i}} & \lambda_{3_{i}}\end{array}\right]^{T}$ o vetor de restrições das forças, $E=\left[\begin{array}{ll}0 & I_{4}\end{array}\right]^{T}$ a matriz de entrada, $\tau_{i}=\left[\begin{array}{ll}\tau_{d_{i}} & \tau_{e_{i}}\end{array}\right]^{T}$ o vetor de torque nas rodas,

$$
\left.C\left(q_{1_{i}}, \dot{q}_{1_{i}}\right)=\left[\begin{array}{ccccc}
0 & 0 & m d \dot{\alpha} \cos \alpha & 0 & 0 \\
0 & 0 & m d \dot{\alpha} \sin \alpha & 0 & 0
\end{array}\right]\right]
$$

a matriz de forças de coriolis e centrípeta, e

$M\left(q_{1_{i}}\right)=\left[\begin{array}{ccccc}m & 0 & m d \sin \alpha_{i} & 0 & 0 \\ 0 & m & -m d \cos \alpha_{i} & 0 & 0 \\ m d \sin \alpha_{i} & -m d \cos \alpha_{i} & I & 0 & 0 \\ 0 & 0 & 0 & I_{\omega} & 0 \\ 0 & 0 & 0 & 0 & I_{\omega}\end{array}\right]$

a matriz de inércia. Os parâmetros $m$ e $I$ são dados por $m=m_{c}+2 m_{\omega}$ e $I=I_{c}+2 m_{\omega}\left(d^{2}+b^{2}\right)+2 I_{m}+m_{c} d^{2}$, sendo $m_{\omega}$ o conjunto da massa da roda e do rotor do motor; $m_{c}$ é a massa da plataforma do robô; $I_{c}$ é o momento de inércia da plataforma do robô em relação ao eixo vertical em $P_{c} ; I_{w}$ é o momento de inércia da roda em relação ao eixo da roda; $I_{m}$ é o momento de inércia da roda em relação ao eixo definido no plano da roda (perpendicular ao eixo da roda). Diferenciando a Eq. (4) em relação ao tempo, substituindo o resultado na expressão dada em (11), e depois multiplicando o lado esquerdo por $S\left(q_{1_{i}}\right)^{T}$, obtém-se

$$
M_{2} \ddot{q}_{2_{i}}+C_{2}\left(\dot{q}_{1_{i}}\right) \dot{q}_{2_{i}}=\tau_{i}
$$

sendo $M_{2}$ uma matriz constante, não-singular, dada por $S^{T}\left(q_{1_{i}}\right) M\left(q_{1_{i}}\right) S\left(q_{1_{i}}\right)$ e $C_{2}\left(\dot{q}_{1_{i}}\right)=C_{2}\left(\dot{\alpha}_{i}\right)$. Na derivação da equação acima utilizou-se o fato que
$S\left(q_{1_{i}}\right)^{T} R\left(q_{1_{i}}\right)^{T}=0$ e $E=\left[\begin{array}{ll}0 & I_{4}\end{array}\right]^{T}$. Adicionando uma perturbação no torque $\delta_{i}=\left[\begin{array}{ll}\delta_{d_{i}} & \delta_{e_{i}}\end{array}\right]^{T}$ e substituindo (5) em (12), tem-se

$$
\ddot{q}_{2_{i}}=\bar{A}\left(\dot{q}_{2_{i}}\right) \dot{q}_{2_{i}}+\bar{B} \tau_{i}+\bar{B} \delta_{i}
$$

sendo $\bar{A}\left(\dot{q}_{2_{i}}\right)=-M_{2}^{-1} C_{2}\left(\dot{q}_{2_{i}}\right)$ e $\bar{B}=M_{2}^{-1}$. Define-se o estado de cada robô como

$$
x_{q_{i}}=\left[\begin{array}{c}
\dot{q}_{2_{i}} \\
q_{2_{i}}
\end{array}\right]=\left[\begin{array}{c}
\dot{\theta}_{d_{i}} \\
\dot{\theta}_{e_{i}} \\
\theta_{d_{i}} \\
\theta_{e_{i}}
\end{array}\right]
$$

e a representação quase-LPV para o controle de acompanhamento de trajetória de cada RMR em formação é dada por

$$
\dot{x}_{q_{i}}=\left[\begin{array}{cc}
\bar{A}\left(\dot{q}_{2_{i}}\right) & 0 \\
I & 0
\end{array}\right] x_{q_{i}}+\left[\begin{array}{l}
I \\
0
\end{array}\right] u_{i}+\left[\begin{array}{c}
\bar{B} \\
0
\end{array}\right] \delta_{i}
$$

sendo

$$
u_{i}=\bar{B} \tau_{i}
$$

Note que o estado é definido com as posições e velocidades angulares das rodas dos RMRs. O controlador baseado na dinâmica proposto neste trabalho considera as posições e velocidades angulares desejadas das rodas, $q_{2_{i}}^{d}$ e $\dot{q}_{2_{i}}^{d}$, respectivamente, para o cálculo do erro de acompanhemento de trajetória. As posições desejadas das rodas, $q_{2_{i}}^{d}$, são calculadas integrando no tempo as velocidades angulares desejadas obtidas pelas equações (3), (9) e (10).

O controle $\mathcal{H}_{\infty}$ com realimentação da saída para sistemas LPV garante que o ganho $\mathcal{L}_{2}$ entre o distúrbio e a saída seja limitado por um nível de atenuação $\gamma$. Para aplicar a técnica de controle apresentada em (Apkarian and Adams, 1998), a equação de acompanhamento de trajetória de cada RMR, (14), deve ser representada de acordo com a seguinte equação

$$
\begin{aligned}
\dot{x}_{q_{i}} & =A\left(\rho_{i}\right) x_{q_{i}}+B_{1}\left(\rho_{i}\right) w_{i}+B_{2}\left(\rho_{i}\right) u_{i}, \\
z_{i} & =C_{1}\left(\rho_{i}\right) x_{q_{i}}+D_{11}\left(\rho_{i}\right) w_{i}+D_{12}\left(\rho_{i}\right) u_{i}, \\
y_{i} & =C_{2}\left(\rho_{i}\right) x_{q_{i}}+D_{21}\left(\rho_{i}\right) w_{i}
\end{aligned}
$$

sendo $\rho_{i}=\left[\rho_{i_{1}}(t), \ldots, \rho_{i_{M}}(t)\right]^{T} \in \mathcal{P}$ o vetor contendo os parâmetros variantes no tempo, $\rho_{i_{k}}(t), k=1, \ldots, M$, que satisfazem $\left|\dot{\rho}_{i_{k}}(t)\right| \leq v_{i_{k}}$, sendo $v_{i_{k}} \geq 0$ os limites da taxa de variação dos parâmetros.

Considere o distúrbio atuando no sistema como uma composição da perturbação no torque, $\delta_{i}$, e pela posições angulares desejadas das rodas, $q_{2_{i}}^{d}$, ou seja, $w_{i}=$ 
$\left[\begin{array}{ll}\delta_{i}^{T} & \left(q_{2_{i}}^{d}\right)^{T}\end{array}\right]^{T}$. A saída medida $z_{i}$ e a saída de controle $y_{i}$ são definidas em termos dos erros de posição, $q_{2_{i}}^{d}-q_{2_{i}}$. Então, (16) pode ser caracterizada pelas seguintes matrizes

$$
\begin{array}{rlrl}
A\left(\rho_{i}\right) & =\left[\begin{array}{cc}
\bar{A}\left(\dot{q}_{2_{i}}\right) & 0 \\
I & 0
\end{array}\right], \quad B_{1}\left(\rho_{i}\right)=\left[\begin{array}{c}
\bar{B} \\
0
\end{array}\right], \\
B_{2}\left(\rho_{i}\right) & =\left[\begin{array}{l}
I \\
0
\end{array}\right], & C_{1}\left(\rho_{i}\right)=\left[\begin{array}{cc}
0 & -I \\
0 & 0
\end{array}\right], \\
C_{2}\left(\rho_{i}\right) & =\left[\begin{array}{ll}
0 & -I
\end{array}\right], & D_{11}\left(\rho_{i}\right)=\left[\begin{array}{ll}
0 & I \\
0 & 0
\end{array}\right], \\
D_{12}\left(\rho_{i}\right) & =\left[\begin{array}{l}
0 \\
I
\end{array}\right], & D_{21}\left(\rho_{i}\right)=\left[\begin{array}{ll}
0 & I
\end{array}\right], \quad D_{22}\left(\rho_{i}\right)=0,
\end{array}
$$

sendo $A_{2}\left(\dot{q}_{2_{i}}\right)$ e $B$ definidas em (13). A dinâmica do controlador é definida como

$$
\left[\begin{array}{c}
\dot{x}_{k_{i}} \\
u_{i}
\end{array}\right]=\left[\begin{array}{cc}
A_{k}\left(\rho_{i}, \dot{\rho}_{i}\right) & B_{k}\left(\rho_{i}, \dot{\rho}_{i}\right) \\
C_{k}\left(\rho_{i}, \dot{\rho}_{i}\right) & D_{k}\left(\rho_{i}, \dot{\rho}_{i}\right)
\end{array}\right]\left[\begin{array}{c}
x_{k_{i}} \\
y_{i}
\end{array}\right] .
$$

Para obter o controlador, é preciso resolver o seguinte conjunto de desigualdades matriciais lineares (DMLs) para $X\left(\rho_{i}\right)$ e $Y\left(\rho_{i}\right)$ minimizando $\gamma$

$$
\begin{aligned}
& {\left[\begin{array}{c|c}
\mathcal{N}_{\mathcal{X}} & 0 \\
\hline 0 & I
\end{array}\right]^{T}\left[\begin{array}{cc|c}
\dot{X}+X A+A^{T} X & X B_{1} & C_{1}^{T} \\
B_{1}^{T} X & -\gamma I & D_{11}^{T} \\
\hline B_{1} & D_{11} & -\gamma I
\end{array}\right] \times } \\
& {\left[\begin{array}{c|c}
\mathcal{N}_{\mathcal{X}} & 0 \\
\hline 0 & I
\end{array}\right]<0, }
\end{aligned}
$$$$
\begin{aligned}
{\left[\begin{array}{c|c}
\mathcal{N}_{\mathcal{Y}} & 0 \\
\hline 0 & I
\end{array}\right]^{T} } & {\left[\begin{array}{cc|c}
-\dot{Y}+Y A^{T}+A Y & Y C_{1}^{T} & B_{1} \\
C_{1} Y & -\gamma I & D_{11} \\
\hline B_{1}^{T} & D_{11}^{T} & -\gamma I
\end{array}\right] \times } \\
& {\left[\begin{array}{c|c}
\mathcal{N}_{\mathcal{Y}} & 0 \\
\hline 0 & I
\end{array}\right]<0, }
\end{aligned}
$$

$$
\left[\begin{array}{cc}
X & I \\
I & Y
\end{array}\right]>0
$$

sendo $\mathcal{N}_{\mathcal{X}}$ e $\mathcal{N}_{\mathcal{Y}}$ projetadas para qualquer base de $\left[\begin{array}{ll}C_{2} & D_{21}\end{array}\right]$ e $\left[\begin{array}{ll}B_{2}^{T} & D_{12}^{T}\end{array}\right]$, respectivamente. Note que embora as matrizes dependam de $\rho_{i}$, essa dependência foi omitida por conveniência. O controlador LPV pode ser encontrado através do seguinte procedimento

- Calcule a solução $D_{k}$ para

$$
\sigma_{\max }\left(D_{11}+D_{12} D_{k} D_{21}\right)<\gamma,
$$

e o conjunto $D_{c l}:=D_{11}+D_{12} D_{k} D_{21}$.
- Calcule as soluções $\widehat{B}_{k}$ e $\widehat{C}_{k}$ para as equações matriciais lineares

$$
\begin{aligned}
& {\left[\begin{array}{ccc}
0 & D_{21} & 0 \\
D_{21}^{T} & -\gamma I & D_{c l}^{T} \\
0 & D_{c l} & -\gamma I
\end{array}\right]\left[\begin{array}{c}
\widehat{B}_{k}^{T} \\
\star
\end{array}\right]=-\left[\begin{array}{c}
C_{2} \\
B_{1}^{T} X \\
C_{1}+D_{12} D_{k} C_{2}
\end{array}\right],} \\
& {\left[\begin{array}{ccc}
0 & D_{12}^{T} & 0 \\
D_{12} & -\gamma I & D_{c l} \\
0 & D_{c l}^{T} & -\gamma I
\end{array}\right]\left[\begin{array}{c}
\widehat{C}_{k} \\
\star
\end{array}\right]=-\left[\begin{array}{c}
B_{2}^{T} \\
C_{1} Y \\
\left(B_{1}+B_{2} D_{k} D_{21}\right)^{T}
\end{array}\right] .}
\end{aligned}
$$

- Calcule

$$
\begin{aligned}
\widehat{A}_{k}= & -\left(A+B_{2} D_{k} C_{2}\right)^{T}+\left[X B_{1}+\widehat{B}_{k} D_{21}\left(C_{1}\right.\right. \\
& \left.\left.+D_{12} D_{k} C_{2}\right)^{T}\right]\left[\begin{array}{cc}
-\gamma I & D_{c l}^{T} \\
D_{c l} & -\gamma I
\end{array}\right]^{-1} \\
& \times\left[\begin{array}{c}
\left(B_{1}+B_{2} D_{k} D_{21}\right)^{T} \\
C_{1} Y+D_{12} \widehat{C}_{k}
\end{array}\right] .
\end{aligned}
$$

- Resolva o problema de fatoração para $N$ e $M$

$$
I-X Y=N M^{T}
$$

- Finalmente, encontre $A_{k}, B_{k}$ e $C_{k}$ dados por

$$
\begin{aligned}
A_{k}= & N^{-1}\left(X \dot{Y}+N \dot{M}^{T}+\widehat{A}_{k}-X\left(A-B_{2} D_{k} C_{2}\right) Y\right. \\
& \left.-\widehat{B}_{k} C_{2} Y-X B_{2} \widehat{C}_{k}\right) M^{-T}, \\
B_{k}= & N^{-1}\left(\widehat{B}_{k}-X B_{2} D_{k}\right), \\
C_{k}= & \left(\widehat{C}_{k}-D_{k} C_{2} Y\right) M^{-T} .
\end{aligned}
$$

Algumas considerações devem ser tomadas para se obter o controlador acima. Primeiro, a solução do conjunto de desigualdades matriciais lineares (DMLs) é um problema de dimensão infinita, uma vez que o vetor de parâmetros $\rho_{i}$ varia continuamente. Para resolver este problema, o espaço de parâmetros $\mathcal{P}$ é dividido em um conjunto de pontos para cada parâmetro, formando-se uma malha de pontos. As DMLs devem ser satisfeitas em todos os pontos desta malha.

Segundo, as matrizes $X\left(\rho_{i}\right)$ e $Y\left(\rho_{i}\right)$ devem variar continuamente com o vetor de parâmetros. A questão é: como expressar estas matrizes em função de $\rho_{i}$ ? Uma maneira de se resolver este problema é definir funções bases para $X\left(\rho_{i}\right)$ e $Y\left(\rho_{i}\right)$ da seguinte forma

$$
\begin{aligned}
X\left(\rho_{i}\right) & =\sum_{p=1}^{P} f_{p}\left(\rho_{i}\right) X_{p} \\
Y\left(\rho_{i}\right) & =\sum_{l=1}^{L} g_{l}\left(\rho_{i}\right) Y_{l}
\end{aligned}
$$


sendo $\left\{f_{p}\left(\rho_{i}\right)\right\}_{p=1}^{P}$ e $\left\{g_{l}\left(\rho_{i}\right)\right\}_{l=1}^{L}$ funções diferenciáveis em $\rho_{i}$. Uma regra prática para escolhermos essas funções é definí-las de maneira semelhante às funções que aparecem na matriz dinâmica do sistema a ser controlado.

A terceira consideração diz respeito às derivadas das variáveis $X\left(\rho_{i}\right)$ e $Y\left(\rho_{i}\right)$ que aparecem nas DMLs. Considera-se neste trabalho que a taxa de variação dos parâmetros é limitada ou, como descrito anteriormente, $\left|\dot{\rho}_{i_{k}}(t)\right| \leq v_{i_{k}}$. Sendo as matrizes $X\left(\rho_{i}\right)$ e $Y\left(\rho_{i}\right)$ descritas como combinações das funções base, as suas derivadas nas DMLs podem ser reescritas como

$$
\begin{gathered}
\dot{X}\left(\rho_{i}\right)=\sum_{k=1}^{M} \pm\left(v_{i_{k}} \sum_{p=1}^{P} \frac{\partial f_{p}}{\partial \rho_{i_{k}}} X_{p}\right) \\
\dot{Y}\left(\rho_{i}\right)=\sum_{k=1}^{M} \pm\left(v_{i_{k}} \sum_{l=1}^{L} \frac{\partial g_{l}}{\partial \rho_{i_{k}}} Y_{l}\right) .
\end{gathered}
$$

$\mathrm{O}$ sinal \pm significa que todas as combinações de sinais positivos e negativos devem ser consideradas para se obter o conjunto de DMLs. Veja (Apkarian and Adams, 1998; Wu et al., 1996) para mais informações sobre a síntese deste tipo de controlador. Observe que com o aumento do número de robôs, o custo computacional para resolver as DMLs aumenta exponencialmente. Entretanto, a implementação dos controladores pode ser realizada dentro de um intervalo de amostragem aceitável e de forma descentralizada.

\section{MODELO MARKOVIANO DOS ROBÔS MÓVEIS}

Nesta seção será apresentada a formulação do controle de formação tolerante a falhas baseado nas metodologias de projeto para sistemas lineares sujeitos a saltos Markovianos. A falha considerada aqui corresponde à perda de um dos robôs da formação, em especial, podese considerar a perda do líder. Após a ocorrência da falha, a dinâmica do robô perdido não é considerada no projeto do controlador baseado em SLSM. Além disso, considera-se que o robô perdido não se tornará, após a falha, um obstáculo para os demais robôs da formação. As metodologias apresentadas neste trabalho podem ser aplicadas em ambientes com obstáculos. Neste caso, a trajetória desejada do líder é alterada para se evitar o obstáculo. Em formações com número elevado de robôs, uma estratégia é dividir a formação em duas formações independentes, cada uma com um líder, cujas trajetórias desejadas evitam os obstáculos.

Os modelos dinâmicos dos robôs móveis em formação são linearizados em torno de pontos definidos na faixa de operação dos robôs móveis, caracterizada a partir dos limites máximos e mínimos das variáveis de posição e velocidade. O modelo Markoviano para este sistema considera os pontos de operação utilizados e a configuração de formação (se há ou não perda de robôs). O estado Markoviano atual é definido pelo ponto de operação atual, pelo número de robôs na formação e pela informação de qual robô é o líder.

Cabe salientar que esta abordagem permite considerar outros aspectos que podem ser úteis para determinadas aplicações como a alternância de líder, falhas de comunicação e obstáculos estáticos e dinâmicos. Tais aspectos serão considerados em trabalhos futuros.

Para obter o sistema linear referente aos pontos de operação, o modelo dinâmico dos RMRs (12), com o acréscimo da perturbação no torque, é representado por

$$
M_{2} \ddot{q}_{2_{i}}+b_{i}=\tau_{i}+\delta_{i}
$$

sendo $b_{i}=C_{2}\left(\dot{q}_{2_{i}}\right) \dot{q}_{2_{i}}$. A linearização de (18) em torno de um ponto de operação $\psi$ com posição $q_{2_{i}}^{\psi}$ e velocidade $\dot{q}_{2_{i}}^{\psi}$, para cada RMR em formação, é dada por

$$
\begin{aligned}
\dot{\bar{x}}_{i} & =A_{i}^{\psi} \bar{x}_{i}+E_{i}^{\psi} \bar{w}_{i}+B_{i}^{\psi} \bar{u}_{i} \\
\bar{z}_{i} & =C_{1_{i}}^{\psi} \bar{x}_{i}+D_{1_{i}}^{\psi} \bar{u}_{i} \\
\bar{y}_{i} & =C_{2_{i}}^{\psi} \bar{x}_{i}+D_{2_{i}}^{\psi} \bar{w}_{i}
\end{aligned}
$$

sendo

$$
A_{i}^{\psi}=\left[\begin{array}{cc}
M_{2}^{-1} k_{p} & -M_{2}^{-1} \frac{\partial b}{\partial \dot{q}_{2_{i}}^{\psi}} \\
0 & I
\end{array}\right]
$$

$$
\begin{gathered}
E_{i}^{\psi}=B_{i}^{j}=\left[\begin{array}{c}
M_{2}^{-1} \\
0
\end{array}\right], \quad C_{1_{i}}^{\psi}=\left[\begin{array}{c}
\alpha_{i} I \\
0
\end{array}\right], \quad D_{1_{i}}^{\psi}=\left[\begin{array}{c}
0 \\
\beta_{i} I
\end{array}\right], \\
C_{2_{i}}^{\psi}=\left[\begin{array}{ll}
0 & I
\end{array}\right], \quad D_{2_{i}}^{\psi}=0, \quad \bar{x}_{i}=\left[\begin{array}{c}
\dot{\theta}_{d_{i}}^{d}-\dot{\theta}_{d_{i}} \\
\dot{\theta}_{e_{i}}^{d}-\dot{\theta}_{e_{i}} \\
\theta_{d_{i}}^{d^{2}}-\theta_{d_{i}} \\
\theta_{e_{i}}^{d^{d}}-\theta_{e_{i}}
\end{array}\right],
\end{gathered}
$$

$\bar{u}_{i}$ a entrada de controle calculada de acordo com o controlador Markoviano apresentado a seguir e $\bar{w}_{i}=\delta_{i} . \alpha_{i}$ e $\beta_{i}$ são ponderações definidas pelo projetista para os erros de acompanhamento de trajetória e para a entrada de controle, respectivamente. O torque aplicado pelos atuadores do $i$-ésimo robô é definido por

$$
\tau_{i}=\left[\begin{array}{ll}
k_{p} & 0
\end{array}\right] \bar{x}_{i}+\bar{u}_{i}
$$

Considera-se neste trabalho que o número de pontos de operação, $\Psi$, é o mesmo para cada uma das formações. 
Considera-se também que as formações resultantes de falha possuem apenas um robô perdido. Ou seja, para uma formação inicial com $N$ robôs, teremos $N+1$ possibilidades de formação. Uma formação com todos os robôs e $N$ formações com um robô perdido. Formações com mais robôs perdidos também podem ser consideradas.

A matriz dinâmica de uma formação inicial com $N$ robôs, para um determinado ponto de operação, é dada por

$$
{ }_{0} A^{\psi}=\left[\begin{array}{ccccc}
A_{1}^{\psi} & \cdots & 0 & \cdots & 0 \\
\vdots & \ddots & \vdots & \cdots & \vdots \\
0 & \cdots & A_{i}^{\psi} & \cdots & 0 \\
\vdots & \cdots & \vdots & \ddots & \vdots \\
0 & \cdots & 0 & \cdots & A_{N}^{\psi}
\end{array}\right]
$$

A matriz dinâmica de uma formação com perda do $i$ ésimo robô é dada por

$$
{ }_{i} A^{\psi}=\left[\begin{array}{ccccc}
A_{1}^{\psi} & \cdots & 0 & \cdots & 0 \\
\vdots & \ddots & \vdots & \cdots & \vdots \\
0 & \cdots & 0 & \cdots & 0 \\
\vdots & \cdots & \vdots & \ddots & \vdots \\
0 & \cdots & 0 & \cdots & A_{N}^{\psi}
\end{array}\right]
$$

As demais matrizes do sistema, ${ }_{i} E^{\psi},{ }_{i} B^{\psi},{ }_{i} C_{1}^{\psi},{ }_{i} C_{2}^{\psi}$, ${ }_{i} D_{1}^{\psi}$ e ${ }_{i} D_{2}^{\psi}$, podem ser construídas de forma similar às matrizes ${ }_{i} A^{\psi}, \psi=1, \cdots, \Psi$.

Considere as coleções de matrizes reais dadas por

$$
\begin{aligned}
A_{\Theta} & =\left({ }_{0} A^{1}, \ldots,{ }_{0} A^{\Psi},{ }_{1} A^{1}, \ldots,{ }_{1} A^{\Psi}, \ldots,{ }_{N} A^{1}, \ldots,{ }_{N} A^{\Psi}\right), \\
E_{\Theta} & =\left({ }_{0} E^{1}, \ldots,{ }_{0} E^{\Psi},_{1} E^{1}, \ldots,{ }_{1} E^{\Psi}, \ldots,{ }_{N} E^{1}, \ldots,{ }_{N} E^{\Psi}\right), \\
B_{\Theta} & =\left({ }_{0} B^{1}, \ldots,{ }_{0} B^{\Psi},_{1} B^{1}, \ldots,{ }_{1} B^{\Psi}, \ldots,{ }_{N} B^{1}, \ldots,{ }_{N} B^{\Psi}\right), \\
C_{1 \Theta} & =\left({ }_{0} C_{1}^{1}, \ldots,{ }_{0} C_{1}^{\Psi}{ }_{1} C_{1}^{1}, \ldots,{ }_{1} C_{1}^{\Psi}, \ldots,{ }_{N} C_{1}^{1}, \ldots,{ }_{N} C_{1}^{\Psi}\right), \\
C_{2 \Theta} & =\left({ }_{0} C_{2}^{1}, \ldots,{ }_{0} C_{2}^{\Psi},_{1} C_{2}^{1}, \ldots,{ }_{1} C_{2}^{\Psi}, \ldots,{ }_{N} C_{2}^{1}, \ldots,{ }_{N} C_{2}^{\Psi}\right), \\
D_{1 \Theta} & =\left({ }_{0} D_{1}^{1}, \ldots,{ }_{0} D_{1}^{\Psi},{ }_{1} D_{1}^{1}, \ldots,{ }_{1} D_{1}^{\Psi}, \ldots,{ }_{N} D_{1}^{1}, \ldots,{ }_{N} D_{1}^{\Psi}\right), \\
D_{2 \Theta} & =\left({ }_{0} D_{2}^{1}, \ldots,{ }_{0} D_{2}^{\Psi},{ }_{1} D_{2}^{1}, \ldots,{ }_{1} D_{2}^{\Psi}, \ldots,{ }_{N} D_{2}^{1}, \ldots,{ }_{N} D_{2}^{\Psi}\right),
\end{aligned}
$$

sendo o conjunto $\Theta$ definido como $\Theta=\{1, \cdots, \Psi(N+$ 1)\}. O número de elementos do conjunto $\Theta$ define o número de estados Markovianos do sistema.

O controle $\mathcal{H}_{\infty}$ com realimentação da saída para SLSM apresentado neste artigo pode ser visto em (Siqueira et al., 2007) e em (de Farias et al., 2000). Considere uma cadeia de Markov homogênea de tempo contínuo, $\theta(t)=\{\theta \in \Theta: t>0\}$, com probabilidade de transição $P_{r}$ definida como:

$P_{r}(\theta(t+\Delta t)=j \mid \theta(t)=i)=\left\{\begin{array}{l}\lambda_{i j} \Delta+o(\delta), \text { se } i \neq j \\ 1+\lambda_{i i} \Delta+o(\delta), \text { se } i=j,\end{array}\right.$

com $i, j \in \Theta, \Delta>0$, e $\lambda_{i j} \geq 0$ a taxa de transição do estado Markoviano $i$ para o estado $j(i \neq j)$, sendo

$$
\lambda_{i i}=-\sum_{j=1, j \neq i}^{\Psi(N+1)} \lambda_{i j} .
$$

A distribuição de probabilidade da cadeia de Markov no tempo inicial é dada por $\mu=\left(\mu_{1}, \ldots, \mu_{\Psi(N+1)}\right)$, sendo $P_{r}(\theta(0)=i)=\mu_{i}$. O sistema linear sujeito a salto Markoviano é dado por

$$
\begin{aligned}
\dot{\bar{x}} & =A_{\theta(t)} \bar{x}+E_{\theta(t)} \bar{w}+B_{\theta(t)} \bar{u}, \\
\bar{z} & =C_{1 \theta(t)} \bar{x}+D_{1 \theta(t)} \bar{u}, \\
\bar{y} & =C_{2 \theta(t)} \bar{x}+D_{2 \theta(t)} \bar{w},
\end{aligned}
$$

$\operatorname{com} \bar{w} \in \mathcal{L}_{2}, E\left(|\bar{x}(0)|^{2}\right)<\infty$.

O controlador dinâmico é dado por

$$
\begin{aligned}
\dot{\bar{x}}_{c} & =A_{c \theta(t)} \bar{x}_{c}+B_{c \theta(t)} \bar{y}, \\
\bar{u} & =C_{c \theta(t)} \bar{x}_{c},
\end{aligned}
$$

sendo $A_{c \theta(t)} \in A_{c \Theta}, B_{c \theta(t)} \in B_{c \Theta}$ e $C_{c \theta(t)} \in C_{c \Theta}$. O problema de controle $\mathcal{H}_{\infty}$ via realimentação de saída para SLSM consiste em encontrar controladores $\left(A_{c \theta}, B_{c \theta}, C_{c \theta}\right)$, com $\theta \in \Theta$, tais que, a norma $\mathcal{H}_{\infty}$ do sistema em malha fechada seja menor que $\gamma$.

Para encontrar estes controladores, o conjunto de DMLs descrito em (19-21) deve ser resolvido. Uma vez obtida a solução para o conjutno de DMLs, os controladores são projetados por

$$
\begin{aligned}
& C_{c \theta}=F_{\theta} Y_{\theta}^{-1} \\
& B_{c \theta}=\left(Y_{\theta}^{-1}-X_{\theta}\right)^{-1} L_{\theta} \\
& A_{c \theta}=\left(Y_{\theta}^{-1}-X_{\theta}\right)^{-1} M_{\theta} Y_{\theta}^{-1}
\end{aligned}
$$

sendo,

$$
\begin{aligned}
M_{\theta}= & -A_{\theta}^{T}-X_{\theta} A_{\theta} Y_{\theta}-X_{\theta} B_{\theta} F_{\theta}-L_{\theta} C_{2 \theta} Y_{\theta} \\
& -C_{1}^{T} \theta\left(C_{1 \theta} Y_{\theta}+D_{1 \theta} F_{\theta}\right)-\gamma^{-2}\left(X_{\theta} E_{\theta}+L_{\theta} D_{2 \theta}\right) E_{\theta}^{T} \\
& -\sum_{j=1}^{\Psi(N+1)} \lambda_{\theta j} Y_{j}^{-1} Y_{\theta} .
\end{aligned}
$$

Embora o custo computacional para se encontrar soluções factíveis para os controladores seja elevado, a implementação destes controladores pode ser realizada dentro 


$$
\begin{gathered}
{\left[\begin{array}{ccc}
A_{\theta}^{T} X_{\theta}+X_{\theta} A_{\theta}+L_{\theta} C_{2 \theta}+C_{2}^{T} L_{\theta}^{T}+C_{1 \theta}^{T} C_{1 \theta}+\sum_{j=1}^{\Psi(N+1)} & \lambda_{\theta j} X_{j} & X_{\theta} E_{\theta}+L_{\theta} D_{2 \theta} \\
E_{\theta}^{T} X_{\theta}+D_{2 \theta}^{T} L_{\theta}^{T} & -\gamma^{2} I
\end{array}\right]<0,} \\
{\left[\begin{array}{ccc}
A_{\theta} Y_{\theta}+Y_{\theta} A_{\theta}^{T}+B_{\theta} F_{\theta}+F_{\theta}^{T} B_{\theta}^{T}+\lambda_{\theta \theta} Y_{\theta}+\gamma^{-2} E_{\theta} E_{\theta}^{T} & Y_{\theta} C_{1 \theta}^{T}+F_{\theta}^{T} D_{1 \theta}^{T} & R_{\theta} Y \\
C_{1 \theta} Y \theta+D_{1 \theta} F_{\theta} & -I & 0 \\
R_{\theta}^{T}(Y) & 0 & S_{\theta}(Y)
\end{array}\right]<0,} \\
{\left[\begin{array}{cc}
Y_{\theta} & I \\
I & X_{\theta}
\end{array}\right]>0 .}
\end{gathered}
$$

sendo

$$
\begin{aligned}
R_{\theta}(Y) & =\left[\sqrt{\lambda_{1 \theta} Y_{\theta}}, \ldots, \sqrt{\lambda_{(\theta-1) \theta} Y_{\theta}}, \sqrt{\lambda_{(\theta+1) \theta} Y_{\theta}}, \ldots, \sqrt{\lambda_{(\Psi(N+1)) \theta} Y_{\theta}}\right] \\
S_{\theta}(Y) & =-\operatorname{diag}\left(Y_{1}, \ldots, Y_{\theta-1}, \ldots, Y_{\theta+1}, \ldots, Y_{\Psi(N+1)}\right) .
\end{aligned}
$$

de um intervalo de amostragem aceitável e de forma descentralizada. A grande dificuldade está no projeto, com o aumento do número de robôs o custo computacional para resolver as DMLs aumenta exponencialmente.

\section{RESULTADOS}

Nesta seção vamos apresentar resultados de simulação para o controle robusto de robôs em formação com alternância de líder baseado no controlador $\mathcal{H}_{\infty}$ não linear apresentado na Seção 4 e para o controle de formação tolerante a falhas baseado no controle Markoviano apresentado na Seção 5. O diagrama de blocos das estratégias de controle propostas neste artigo é mostrado na Figura 4.

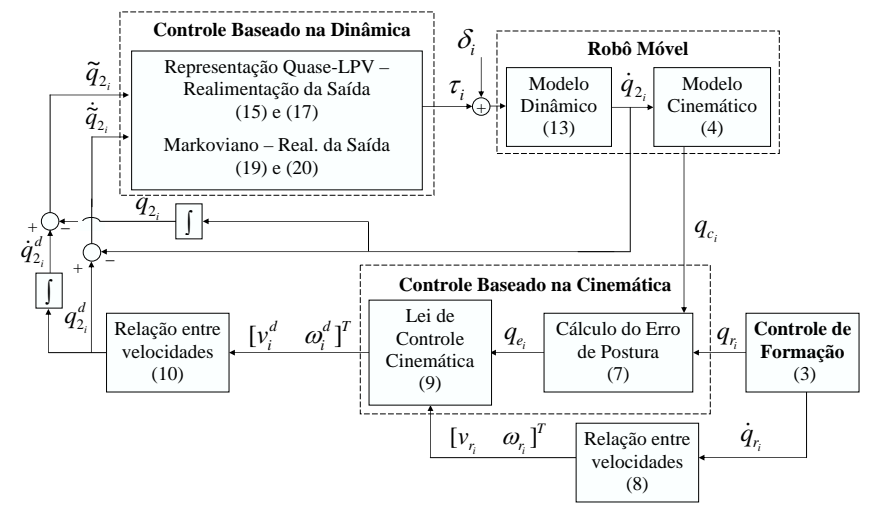

Figura 4: Diagrama de blocos detalhado das estratégias de controle.

Inicialmente, consideram-se seis RMRs em formação. A Fig. 5 mostra seis possíveis dígrafos, um para cada possiblidade de alternância dos robôs na liderança da formação, ou seja, para cada robô na liderança tem-se uma
$L_{\Gamma}$. Observe que os círculos em vermelho correspondem ao robô líder da formação. Foi considerada a seguinte formação para os RMRs

$$
\begin{aligned}
& \left(h_{p}\right)_{1}=\left[\begin{array}{ll}
6 & 0
\end{array}\right], \quad\left(h_{p}\right)_{2}=\left[\begin{array}{ll}
3 & 2
\end{array}\right], \quad\left(h_{p}\right)_{3}=\left[\begin{array}{ll}
3 & -2
\end{array}\right], \\
& \left(h_{p}\right)_{4}=\left[\begin{array}{ll}
0 & 4
\end{array}\right], \quad\left(h_{p}\right)_{5}=\left[\begin{array}{ll}
0 & 0
\end{array}\right], \quad\left(h_{p}\right)_{6}=\left[\begin{array}{ll}
0 & -4
\end{array}\right] \text {. }
\end{aligned}
$$
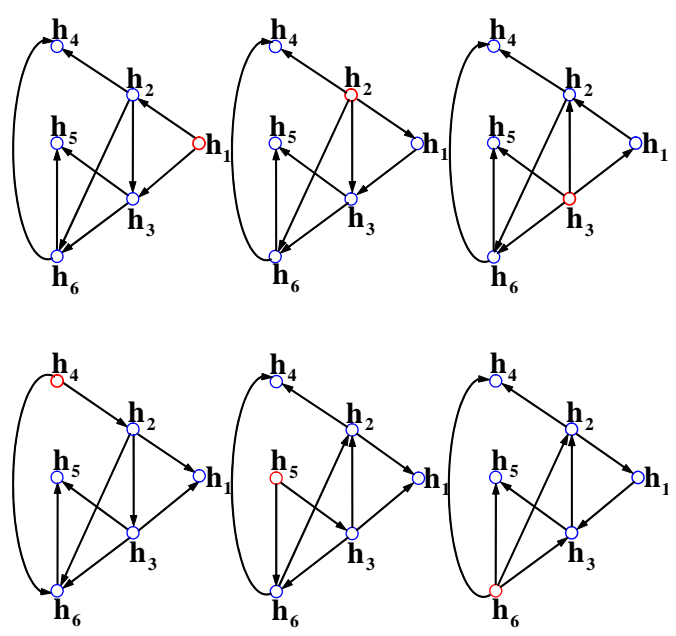

Figura 5: Dígrafos para cada um dos líderes da formação.

Os robôs serão denominados conforme sua posição na formação, ou seja, o Líder 1 será o robô que está na posição $h_{1}$ e assim por diante. A matriz de realimentação $F_{v e h}$ aplicada a todos os robôs da formação é dada por

$$
F_{\text {veh }}=\left[\begin{array}{cccc}
-310 & -400 & 0 & 0 \\
0 & 0 & -310 & -400
\end{array}\right] \text {. }
$$

Para o controlador baseado na cinemática, os ganhos 
foram definidos como $K_{x}=7, K_{y}=52, K_{\alpha}=27$. Os valores destes ganhos foram escolhidos heuristicamente buscando obter uma resposta rápida, porém suave, da trajetória. As trajetórias de referência, $x_{r_{i}}$ e $y_{r_{i}}$, são dadas pela Eq. (3) com condições iniciais

$$
\begin{aligned}
& \left(x_{c_{1}}(0), y_{c_{1}}(0), \alpha_{c_{1}}(0)\right)=(0,4,0), \\
& \left(x_{c_{2}}(0), y_{c_{2}}(0), \alpha_{c_{2}}(0)\right)=(0,6,0), \\
& \left(x_{c_{3}}(0), y_{c_{3}}(0), \alpha_{c_{3}}(0)\right)=(0,2,0), \\
& \left(x_{c_{4}}(0), y_{c_{4}}(0), \alpha_{c_{4}}(0)\right)=(0,14,0), \\
& \left(x_{c_{5}}(0), y_{c_{5}}(0), \alpha_{c_{5}}(0)\right)=(0,12,0), \\
& \left(x_{c_{6}}(0), y_{c_{6}}(0), \alpha_{c_{6}}(0)\right)=(0,10,0) .
\end{aligned}
$$

Os seguintes parâmetros nominais são considerados para todos os robôs, eles correspondem aos parâmetros do robô móvel construído em nosso laboratório (Inoue et al., 2009): $m_{\omega}=0,075(\mathrm{~kg}), m_{c}=0,597(\mathrm{~kg})$, $a=0,17(\mathrm{~m}), b=0,065(\mathrm{~m}), d=0,01(\mathrm{~m}), r=0,028$ $(\mathrm{m}), I_{c}=0,0023\left(\mathrm{~kg} \cdot \mathrm{m}^{2}\right), I_{\omega}=0,00038\left(\mathrm{~kg} \cdot \mathrm{m}^{2}\right) \mathrm{e}$ $I_{m}=3,7 \times 10^{-7}\left(\mathrm{~kg} . \mathrm{m}^{2}\right)$.

Para o controlador $\mathcal{H}_{\infty}$ via representação quase-LPV, o espaço de variação dos parâmetros $\rho_{i}, \mathcal{P}$, definido como

$$
-\pi \leq \dot{\tilde{\theta}}_{d_{i}} \leq \pi(\mathrm{rad} / \mathrm{s}) \quad e \quad-\pi \leq \dot{\tilde{\theta}}_{e_{i}} \leq \pi(\mathrm{rad} / \mathrm{s}),
$$

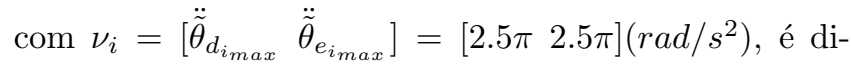
vidido em $L=3$ pontos para cada parâmetro. Note que os parâmetros selecionados correspondem aos erros de velocidade das rodas direita e esquerda de cada robô. As funções base foram selecionadas de modo a apresentar um comportamento semelhante ao sistema a ser controlado $f_{1}\left(\rho_{i}\right)=g_{1}\left(\rho_{i}\right)=10^{6}, g_{2}=10^{11} \cos \dot{\tilde{\theta}}_{d_{i}} \mathrm{e}$ $g_{3}\left(\rho_{i}\right)=10^{9} \operatorname{sen} \dot{\tilde{\theta}}_{e_{i}}$, sendo $X(\theta)$ e $Y(\theta)$ definidas como segue

$$
\begin{aligned}
X\left(\rho_{i}\right) & :=X_{0} f_{1}\left(\rho_{i}\right) \\
Y\left(\rho_{i}\right) & :=Y_{0} g_{1}\left(\rho_{i}\right)+Y_{1} g_{2}\left(\rho_{i}\right)+Y_{2} g_{3}\left(\rho_{i}\right) .
\end{aligned}
$$

O melhor nível de atenuação encontrado foi $\gamma=2.61$. Para testar a robustez dos controladores, distúrbios externos foram introduzidos nos torques das rodas da seguinte forma

$$
\begin{aligned}
& \delta_{d_{i}}=1 e^{-(t-4)^{4}} \operatorname{sen}(1.3 \pi t), \\
& \delta_{e_{i}}=-1 e^{-(t-4)^{4}} \operatorname{sen}(1.3 \pi t)
\end{aligned}
$$

A simulação do controlador $\mathcal{H}_{\infty}$ com realimentação da saída foi realizada para os seis robôs móveis, que se alternaram na liderança da formação (sendo o robô 1 o líder inicial, o robô 6 o líder intermediário e o robô 4 o líder final) durante a trajetória, Fig. 6. A sequência de liderança é definida aleatoriamente segundo uma matriz de probabilidade uniforme para todos os robôs da formação, ou seja, qualquer robô pode assumir a liderança com a mesma probabilidade (no exemplo considerado, esta probabilidade é $1 / 6$ ). Note que, para fins de simulação, foram realizadas duas alternâncias de líder dentro do intervalo de tempo considerado, em espaços regulares da trajetória, respectivamente, a um terço e dois terços desta. O projetista também pode definir uma função densidade de probabilidades que descreve os instantes de tempo em que há maior probabilidade de ocorrer a alternância de líder.

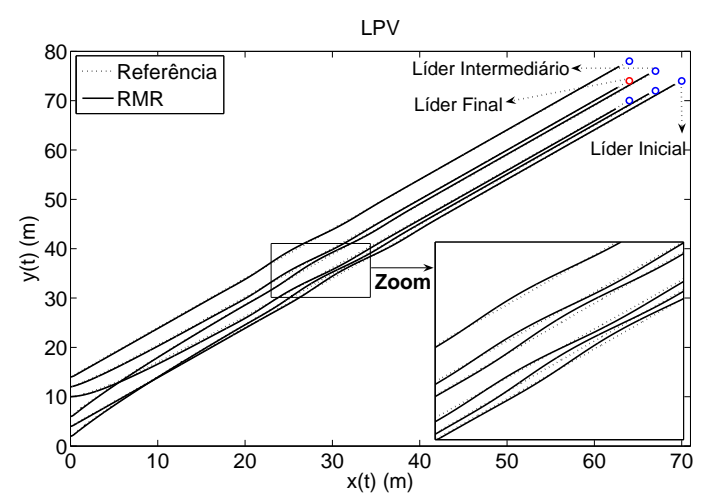

Figura 6: Controle quase-LPV com realimentação da saída para os RMRs em formação. A região em destaque mostra o momento no qual o distúrbio foi aplicado.

O erros de direção dos robôs durante a trajetória com alternância de líder são mostrados na Figura 7. Observe que os distúrbios foram rejeitados adequadamente. Nas figuras 8 e 9 são mostrados os distúrbios e torques, respectivamente, aplicados nas rodas atuadas.

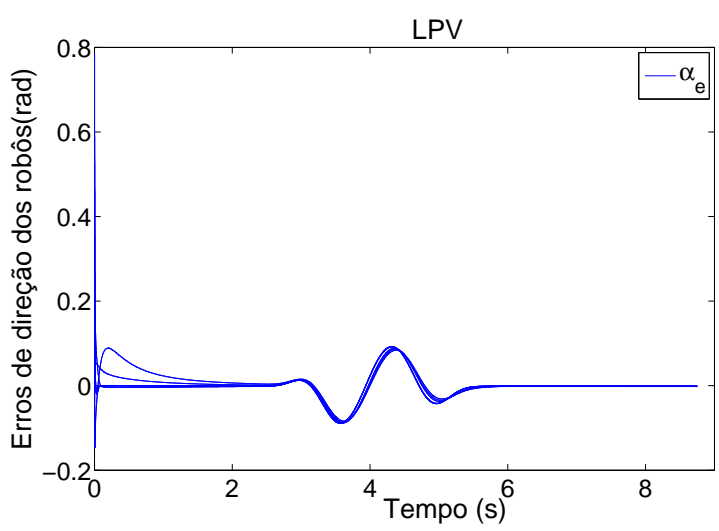

Figura 7: Erros de direção dos robôs móveis.

Para o controle tolerante a falhas, foi considerada como 


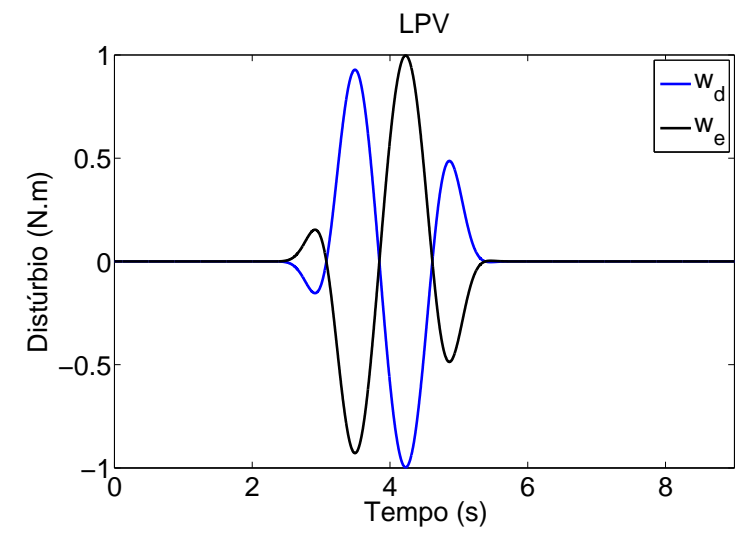

Figura 8: Distúrbios aplicados nas rodas direita e esquerda dos robôs.

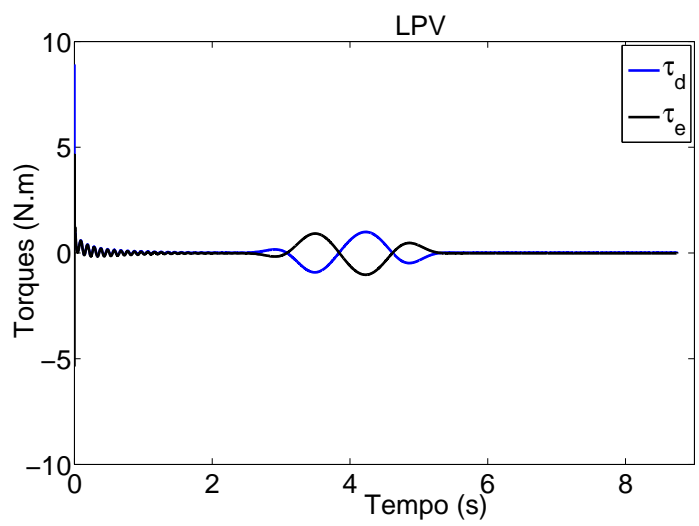

Figura 9: Torques aplicados nas rodas direita e esquerda dos robôs.

falha a remoção do líder de uma formação composta por três robôs $(N=3)$. Assim, o sistema inicia o movimento coordenado com um conjunto de três robôs e durante o movimento o robô líder inicial é removido. Imediatamente um segundo robô assume a liderança até que o movimento seja finalizado. Neste experimento não está sendo considerada a alternância aleatória de líder. Considera-se também que um ponto de linearização em $1 \mathrm{rad} / \mathrm{s}$ caracterize ambos os cenários pré e pós falha para calcular as respectivas matrizes dinâmicas do sistema linear, ou seja, $\Psi=1$. Portanto, temos 2 estados Markovianos, um para cada formação. Seguindo a notação apresentada na Seção 5 , o conjunto de matrizes dinâmicas do modelo Markoviano é dado por $A_{\Theta}=\left({ }_{0} A^{1},{ }_{1} A^{1},\right)$, sendo que 0 à esquerda de $\mathrm{A}$ significa que o movimento coordenado evolui inicialmente com todos os robôs e 1 significa que houve uma falha e o robô 1 foi excluído da formação. Para esse modelo Markoviano $\Theta=\{1,2\}$.
As taxas de transição entre os estados Markovianos são agrupadas em uma matriz de taxa de transição $\Lambda$ de dimensão 2 x 2 . A matriz $\Lambda$ é particionada nas seguintes submatrizes

$$
\Lambda=\left[\begin{array}{cc}
\Lambda_{p_{1}} & \Lambda_{f} \\
\Lambda_{0} & \Lambda_{p_{2}}
\end{array}\right]
$$

As submatrizes $\Lambda_{p_{i}}$ mostram as relações entre os pontos de operação, e a submatriz diagonal $\Lambda_{f}$ determina a probabilidade de ocorrência de falha. Depois da falha ter ocorrido o sistema muda para a segunda linha de $\Lambda$, e $\Lambda_{0}$ mostra que a formação inicial não pode ser recuperada. Para o problema em questão, a matriz de taxa de transição $\Lambda$ foi selecionada como sendo

$$
\begin{aligned}
\Lambda_{p_{1}} & =-0.6, & \Lambda_{p_{2}} & =0, \\
\Lambda_{f} & =0.6, & \Lambda_{0} & =0 .
\end{aligned}
$$

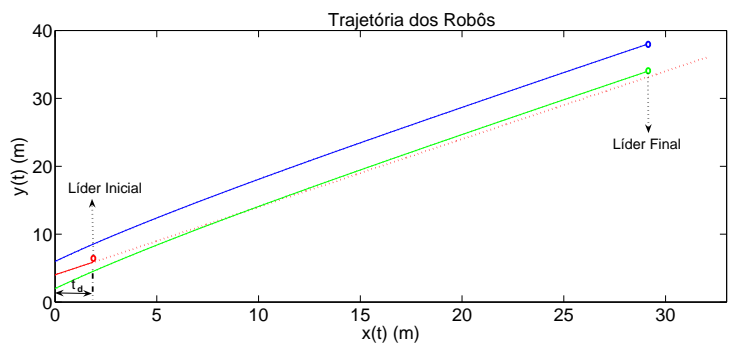

Figura 10: Trajetória dos robôs com controlador Markoviano.

A Figura 10 mostra os resultados experimentais da trajetória efetuada pela formação utilizando o controlador Markoviano via realimentação de saída. Inicialmente o líder da formação era o robô representado pelo círculo em vermelho, e, em $t_{d}=0,5 \mathrm{~s}$ este robô foi removido e a falha detectada. A partir deste instante, o robô 2 assumiu a liderança da formação (indicado na figura pelo círculo verde). Note que neste experimento não há uma alternância aleatória de líder, e sim, uma troca devido à falha do robô 1 . Após a falha, o robô 1 não influencia a dinâmica dos demais robôs, pois ele não pertence à nova formação. As linhas tracejadas na Figura 10 denotam as trajetórias desejadas dos RMRs e a linha contínua a trajetória real. Também é mostrado na Figura (11) o erro de direção do robô 1 durante a trajetória. Das simulações obtidas, observa-se que após a ocorrência da falha o sistema mantém a estabilidade com os robôs em movimento coordenado durante a reconfiguração do controle.

\section{CONCLUSÕES}

Neste artigo foram propostas duas estratégias de controle para movimentos coordenados de robôs móveis com 


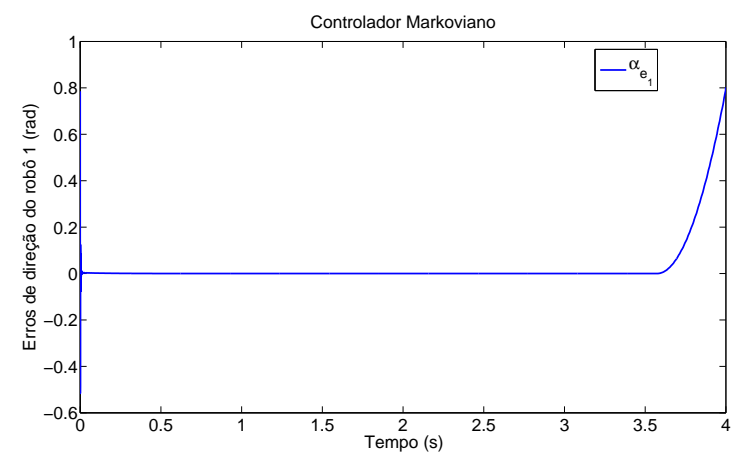

Figura 11: Erro de direção do robô 1 com controlador Markoviano.

rodas, uma robusta e outra tolerante a falhas. Inicialmente, considerou-se uma formação com alternância de líder sujeita a distúrbios externos. O controle robusto $\mathcal{H}_{\infty}$ não linear por realimentação da saída, em conjunto com os controles de formação e cinemático, garante a estabilidade da formação considerando as características dinâmicas dos robôs móveis. A segunda estratégia considera que a formação pode sofrer a perda de robôs, inclusive do líder. Neste caso, foi proposto um controle tolerante a falhas baseado na teoria de sistemas lineares sujeitos a saltos Markovianos. Novamente, o controlador $\mathcal{H}_{\infty}$ por realimentação da saída para SLSM garante a estabilidade global do sistema mesmo com a perda de robôs da formação. Os aspectos principais das duas metodologias propostas foram considerados nos exemplos apresentados, robustez a distúrbios externos e tolerância a falhas. Entretanto, há uma infinidade de situações nas quais a metodologia proposta neste artigo precisa ser aperfeiçoada para funcionar com eficiência, por exemplo quando ocorrem falhas de comunicação ou quando aparecem obstáculos de maneira aleatória. Esses problemas serão tratados futuramente.

\section{AGRADECIMENTOS}

Este trabalho contou com o apoio FAPESP através do processo 06/02303-7.

\section{REFERÊNCIAS}

Apkarian, P. and Adams, R. (1998). Advanced gain-scheduling techniques for uncertain systems, IEEE Transactions on Control Systems Technology 6(1): $21-32$.

Brualdi, R. and Ryser, H. (1991). Combinatorial Matrix Theory, Cambridge University Press, USA.
Chaimowicz, L., Kumar, V. and Campos, M. F. M. (2004). A paradigm for dynamic coordination of multiple robots, Autonomous Robots 17(1): 7-21.

Coelho, P. and Nunes., U. (2003). Lie algebra application to mobile robot control: A tutorial, Robotica 21: 483-493.

de Farias, D. P., Geromel, J. C., do Val, J. B. R. and Costa, O. L. V. (2000). Output feedback control of Markov jump linear systems in continuoustime, IEEE Transactions on Automatic Control 45(5): 944-949.

Desai, J., Ostrowski, J. and Kumar., V. (1998). Controlling formations of multiple robots, Proceedings of the IEEE International Conference on Robotics and Automation (ICRA98), Leuven, Belgium, pp. 2864-2869.

Fax, J. and Murray., R. (2004). Information flow and cooperative control of vehicle formations, IEEE Transactions on Automatic Control 49(9): 1465-1476.

Inoue, R. S., Siqueira, A. A. G. and Terra, M. H. (2009). Experimental results on the nonlinear $\mathcal{H}_{\infty}$ control via quasi-LPV representation and Game Theory for wheeled mobile robots, Robotica 27 (4): 547-553.

Kanayama, Y., Kimura, Y., Miyazaki, F. and Noguchi., T. (1990). A stable tracking control method for an autonomous mobile robots, in Proc. IEEE International Conference on Robotics and Automation pp. 384-389.

Laferriere, G., Caughman, J. and Willians, A. (2004). Graph theoretic methods in the stability of vehicle formations, Proceeding of American Control Conference pp. 3724-3729.

Lafferriere, G., Williams, A., Caughman, J. and Veerman., J. J. P. (2005). Descentralized control of vehicle formations, Systems and Control Letters 54(9): 899-910.

Olfati-Saber, R. and Murray, R. M. (2002). Distributed sctrutural stabilization and tracking for formations of dynamic multiagents, Procedings of IEEE Conference on Decision and Control pp. 209-215.

Pereira, G. A. S., Kumar, V. and Campos, M. F. M. (2008). Closed loop motion planning of cooperating mobile robots using graph connectivity, Robotics and Autonomous Systems 56(4): 373-384.

Siqueira, A. A. G., Terra, M. H. and Buosi, C. (2007). Fault-tolerant robot manipulators based on outputfeedback $\mathcal{H}_{\infty}$ controllers, Journal of Robotics and Autonomous Systems 55(10): 785-794. 
Tanner, H., Pappas, G. and Kumar., V. (2004). Leaderto-formation stability, IEEE Transactions on Robotics and Automation 20(3): 443-455.

Veerman, J. J. P., Lafferriere, G., Caughman, J. S. and Williams, A. (2005). Flocks and formations, Journal of Statistical Physics 121(5-6): 901-936.

Williams, A., Lafferriere, G. and Veerman, J. (2005). Stable motions of vehicles formations, Proceedings of the IEEE Conference on Decision and Control and the European Control Conference pp. 12-15.

Wu, F., Yang, X. H., Packard, A. and Becker., G. (1996). Induced $\mathrm{L}_{2}$ norm control for LPV systems with bounded parameter variation rates, International Journal of Robust and Nonlinear Control 6(910): 983-998. 